\title{
Enteric Viruses in a Large Waterborne Outbreak of Acute Gastroenteritis in Finland
}

\author{
L. Maunula $\cdot$ P. Klemola $\cdot$ A. Kauppinen $\cdot$ K. Söderberg $\cdot$ T. Nguyen $\cdot$ \\ T. Pitkänen - S. Kaijalainen · M. L. Simonen · I. T. Miettinen · \\ M. Lappalainen - J. Laine $\cdot$ R. Vuento $\cdot$ M. Kuusi $\cdot$ M. Roivainen
}

Published online: 11 February 2009

(C) Springer Science+Business Media, LLC 2009

\section{Erratum to: Food Environ Virol (2009) 1:31-36}

\section{DOI 10.1007/s12560-008-9004-3}

The correct affiliation for P. Klemola, T. Nguyen, S. Kaijalainen, M. L. Simonen, J. Laine, M. Kuusi, and M. Roivainen is as follows:

National Public Health Institute, Helsinki, Finland

The online version of the original article can be found under doi:10.1007/s12560-008-9004-3.

L. Maunula $(\varangle) \cdot$ K. Söderberg Department of Food and Environmental Hygiene, Faculty of Veterinary Medicine, University of Helsinki, P.O. Box 66, 00014 Helsinki, Finland

e-mail: leena.maunula@helsinki.fi

P. Klemola $\cdot$ T. Nguyen $\cdot$ S. Kaijalainen

M. L. Simonen · J. Laine · M. Kuusi · M. Roivainen

National Public Health Institute, Helsinki, Finland

A. Kauppinen · T. Pitkänen · I. T. Miettinen

National Public Health Institute, Kuopio, Finland

M. Lappalainen

Laboratory Division (HUSLAB), Department of Virology,

Helsinki University Central Hospital, Helsinki, Finland

J. Laine

Tampere University Hospital, Tampere, Finland

R. Vuento

Centre for Laboratory Medicine,

Tampere University Hospital, Tampere, Finland 